\author{
Feng-Feng Pan \\ Shanghai Sixth Peoples Hospital \\ Qi Huang \\ Huashan Hospital Fudan University \\ Ying Wang \\ Shanghai Sixth Peoples Hospital \\ Yi-Fan Wang \\ Shanghai Sixth Peoples Hospital \\ Yi-Hui Guan \\ Huashan Hospital Fudan University \\ Fang Xie \\ Huashan Hospital Fudan University \\ Qihao Guo ( $\square$ qhguo@sjtu.edu.cn ) \\ Shanghai Jiao Tong University
}

$\angle$ Research Square

Preprints are preliminary reports that have not undergone peer review.

They should not be considered conclusive, used to inform clinical practice, or referenced by the media as validated information.

\section{Changes of plasma $A \beta$ and $t$-tau along with 18F-florbetapir PET in a cohort of cognitive decline}

\section{Research}

Keywords: Alzheimer's disease (AD), Plasma amyloid- $\beta$, Plasma t-tau, Simoa, Amyloid- $\beta$ PET

Posted Date: June 17th, 2021

DOI: https://doi.org/10.21203/rs.3.rs-615251/v1

License: (c) (i) This work is licensed under a Creative Commons Attribution 4.0 International License. Read Full License 


\section{Abstract \\ Background}

Blood based biomarkers for Alzheimer's disease (AD) are becoming increasingly promising. Although plasma amyloid- $\beta$ (A $\beta$ ) and total tau (ttau) showed a potential ability in identifying cerebral amyloid deposition and AD. Comparisons of these plasma biomarkers along with $A \beta$ PET in a cohort of normal controls (NC), subjective cognitive decline (SCD), objectively-defined subtle cognitive decline (Obj-SCD), mild cognitive impairment $(\mathrm{MCl})$ and $A D$ remained lacking.

\section{Methods}

A total of 407 individuals aged from 40 to 90 years old were recruited, including 76 of NC, 77 of SCD, 61 of obj-SCD, 92 of MCl and 101 of AD. Plasma $A \beta 40, A \beta 42$ and t-tau were examined via single-molecule array (Simoa) immunoassay. A subset of 132 individuals underwent cerebral amyloid scans with $18 \mathrm{~F}$-florbetapir PET. Comparisons of plasma t-tau, $A \beta 40, A \beta 42$ and $A \beta 42 / A \beta 40$ ratio were conducted between different diagnostic groups and cerebral $A \beta$ burdens. Pearson correlation analysis was used to evaluate the correlation between $A \beta 42, A \beta 42 / A \beta 40$ ratio and 18F-florbetapir SUVR. Receiver operating characteristic (ROC) curve analyses were carried out to evaluate the capacity of plasma biomarkers in identifying high brain $A \beta$ burden and diagnosis of $A D$.

\section{Results}

Plasma $A \beta 42$ was significantly higher in SCD and obj-SCD than NC, MCl and AD. Plasma A 340 was significantly higher in SCD and obj-SCD than $N C$ and $A D$. The lowest levels of plasma $A \beta 42$ and $A \beta 42 / A \beta 40$ ratio were found in $A D$. No significant difference of plasma t-tau was found among groups. Plasma $A \beta 42$ and $A \beta 42 / A \beta 40$ ratio were inversely correlated with $18 F$-florbetapir $S U V R(r=-0.272, P=0.003 ; r=-0.211$, $P$ $=0.021$ respectively). $A \beta 42 / A \beta 40$ ratio performed well in predicting high brain $A \beta$ burden (area under the curve, $A U C=0.762$ ). Plasma $A \beta 42$ and $A \beta 42 / A \beta 40$ ratio had acceptable diagnostic accuracy for $A D(A U C=0.714$ and 0.706 respectively), even in $A \beta-P E T(+)$ individuals $(A U C=$ 0.728 and 0.808 respectively).

\section{Conclusions}

Plasma $A \beta 40$ and $A \beta 42$ measured by Simoa immunoassay showed a significantly bidirectional trend of initially increasing from NC to SCD and obj-SCD, and then declining to $\mathrm{MCl}$ and $\mathrm{AD}$. In addition, plasma $A \beta$ was significantly correlated with $18 \mathrm{~F}$-florbetapir PET SUVR and showed potential value in predicting cerebral $A B$ deposition and risk of $A D$.

\section{Background}

Deposition of amyloid- $\beta$ (A $\beta$ ) plaques and neurofibrillary tangles (NFTs) of tau fibrils are still the primary neuropathological hallmarks of Alzheimer's disease (AD)(1). Though the examination of cerebrospinal fluid (CSF) and PET imaging provide the most reliable AD-related biomarkers of $A \beta$ and tau in-vivo $(2,3)$, the invasive sampling procedure of CSF and high cost of PET image hinder their application in clinical trials and population screening, especially in the preclinical stages of AD. Blood-based biomarkers have the advantages of less invasive, more cost effective and easy to handle, potentially enabling its usage in population screening and follow-up. Owing to the emergence of ultrasensitive technologies such as Immunoprecipitation-mass spectrometry (IP-MS)(4) and single molecule array (Simoa)(5), measuring AD-related biomarkers in blood becomes increasingly promising. With these high sensitivity measurements, previous studies showed that plasma $A \beta$ was significantly correlated with $\operatorname{CSF}(6-8)$, and had a high performance in predicting cerebral amyloid deposition (8-10). Consistent with the association between low plasma $A \beta$ and increasing cerebral amyloid deposition, plasma $A \beta$ isoforms and $A \beta 42 / A \beta 40$ ratio were significantly lower in patients with $A D(6,11,12)$. Even in the dementia-free populations, lower plasma levels of $A \beta 42$ or $A \beta 42 / A \beta 40$ ratio are associated with steeper rate of cognitive decline or increased risk of $A D$ and dementia(13-15).

Currently, even though the significant correlations between low plasma $A \beta$ and $A D$ were identified, confusing findings still remain. For example, in the large sample cohort of AIBL, plasma concentrations of $A \beta 42$ and $A \beta 40$ measured by ELISA showed an increasing trend from health controls to mild cognitive impairment (MCl), but decreased from $\mathrm{MCl}$ to $A D(16)$. Similarly, a population based cross-sectional study found that plasma $A \beta 42$ and $A \beta 42 / A \beta 40$ ratio in the possible cognitive impairment were higher than both the normal cognition group and probable cognitive impairment group(17). A recent longitudinal study showed that plasma $A \beta 42$ levels increased gradually before the stage of $\mathrm{MCl}$ and decreased just prior to clinical $\mathrm{AD}$ onset(18). Taken together, these data suggest a dynamic, nonlinear character of plasma amyloid- $\beta$ over the course of cognitive decline. However, this trend of plasma amyloid- $\beta$ was mostly derived from the cognitive status of $\mathrm{MCl}$ and 
dementia, but not took into account the earlier status such as subjective cognitive decline (SCD) and objectively-defined subtle cognitive decline (Obj-SCD), which may lead to not accurately reflect the evolution of plasma biomarkers across a complete spectrum of cognitive decline.

The aims of this study were to examine plasma $A \beta 42, A \beta 40$ and t-tau with Simoa immunoassay in a cohort of cognitive decline, explore their evolution among normal controls $(\mathrm{NC}), \mathrm{SCD}, \mathrm{Obj}-\mathrm{SCD}, \mathrm{MCl}$ and $\mathrm{AD}$, and investigate their abilities in identifying cerebral amyloid deposition and diagnosis of $A D$.

\section{Methods}

\section{Study participants}

In this monocentric retrospective cohort study, a total of 407 individuals were enrolled from Sixth People's Hospital, Shanghai, China, from January 2019 to January 2021. Participants were aged 40 to 90 years, educated more than one year. Individuals with a history of significant neurologic disease, psychiatric disorders, alcoholism, drug abuse and head trauma were excluded. Routine laboratory tests and cranial MRI scanning were carried out to preclude relevant diseases which may be adversely affecting cognitive function, such as abnormalities in folic acid, vitamin B12, thyroid function, cerebral infarction, subdural hematomas, hydrocephalus, intracranial tumors and infections. All the participants underwent a battery of standardized neuropsychological tests and examined plasma $A \beta 42, A \beta 40$ and t-tau via Simoa immunoassay. A total of 132 participants underwent 18F-florbetapir PET scan within 3 months after blood sampling. Written informed consent was obtained from all the participants or their caregivers. The ethics committee of Shanghai Jiao Tong University Affiliated Sixth People's Hospital approved this study.

\section{Neuropsychological Assessment}

General cognitive performance was assessed by Mini-Mental State Examination (MMSE)(19), Montreal Cognitive Assessment-Basic (MoCABC)(20) and Chinese version of Addenbrooke's Cognitive Examination-III (ACE-III-CV)(21). Global functional status was assessed by Everyday Cognition (ECOG)(22) and Functional Assessment Questionnaire (FAQ)(23). In addition, a battery of standardized neuropsychological tests including six neuropsychological indexes was carried out: Auditory Verbal Learning Test (AVLT)(24) 30-minute delayed free recall and AVLT recognition for memory, Boston Naming Test (BNT)(25) and Animal Verbal Fluency Test (AFT)(26) for language, Shape Trail Test Part A and B (STT-A, STT-B)(27) for executive function.

\section{Cognitive groups}

The clinical diagnosis of AD was made by experienced neurologists according to the National Institute on Aging and Alzheimer's Association (NIA-AA) criteria for probable AD dementia(28). In the non-dementia participants, $\mathrm{MCl}$ and Obj-SCD was classified according to the criteria proposed by Jak and Bondi(29). Diagnosis of $\mathrm{MCl}$ was given if the participant met one of the following criteria: (1) impaired scores (defined as $>1$ standard deviation (SD) below the age-corrected normative mean) on two of the six neuropsychological indexes in the same cognitive domain; (2) impaired scores in each of the three cognitive domains; (3) FAQ score $\geq 9$. Participants were classified as Obj-SCD according to one of the following criteria: (1) impaired scores on two of the six neuropsychological indexes in the different cognitive domains; (2) impaired scores on one of the six neuropsychological indexes; (3) FAQ score of 6-8. Participants with self-reported experiences of cognitive decline but not met the criterion for $\mathrm{MCl}$ and Obj-SCD were classified as SCD according to the conceptual framework proposed by the Subjective Cognitive Decline Initiative (SCD-I) working group(30). Normal controls were recruited from caregivers or through advertisements, with no cognitive complaint and impairment determined by neuropsychological tests.

\section{Blood processing and measurements of plasma $A \beta 42, A \beta 40, t$-tau}

Blood samples were centrifuged at $500 \mathrm{~g}$ for 5 minutes at $4{ }^{\circ} \mathrm{C}$ to collect plasma. Then plasma was immediately aliquoted into ultra-low adsorption tubes (AXYGEN MCT-150-L-C) on ice and stored at $-80^{\circ} \mathrm{C}$ refrigerator. Before the test, plasma samples were transferred from the refrigerator to ice plate for 30 minutes, and then centrifuged at $10,000 \mathrm{~g}$ for $5 \mathrm{~min}$ at $4{ }^{\circ} \mathrm{C}$. Total tau measurement was performed using Neurology 4-Plex A kit (Quanterix 102153), Aß42 and Aß40 were performed using Neurology 3-Plex A assay (Quanterix 101995) on the Simoa HD-1 platform (Quanterix)(31). Reagent pretreatment and sample loading were carried out according to the instructions of the manufacture. In brief, $350 \mu \mathrm{L}$ pre-diluted calibrator reagent $(0-100 \mathrm{pg} / \mathrm{mL}$ for t-tau, $0-50 \mathrm{ng} / \mathrm{mL}$ for $A \beta 42$ and $0-150 \mathrm{ng} / \mathrm{mL}$ for A $\mathrm{A} 40$ ) and $320 \mu \mathrm{L}$ plasma sample (1:4 diluted) were loaded on the plate. Concentrations of each marker $(\mathrm{pg} / \mathrm{mL})$ were calculated from the calibration curve. All the biomarker measurements were performed by laboratory technicians who were blinded to the clinical data.

\section{F-florbetapir PET acquisition and analysis}


Amyloid PET images were obtained from a PET/CT system (Biograph mCT Flow PET/CT, Siemens, Erlangen, Germany) at the PET center of Huashan hospital, Fudan University. Cerebral amyloid PET scans were carried out 50min after the intravenous injection of 7.4 $\mathrm{MBq} / \mathrm{kg}(0.2 \mathrm{mCi} / \mathrm{kg})$ florbetapir and lasted for $20 \mathrm{~min}$. PET images were reconstructed using filtered back projection algorithm with corrections for decay, normalization, dead time, photon attenuation, scatter and random coincidences. PET images were coregistered to the individual structural MRI and partial volume error correction (PVC) was performed using the Muller-Gartner methods(32, 33), followed by spatially normalized in the Montreal Neurological Institute (MNI) template and Gaussian smoothing. Standard uptake value ratios (SUVR) were calculated for each cortical region of interest (ROI) relative to cerebellar crus. Global SUVR scores were calculated by weighted averaging of these ROIs. Subjects were diagnosis as 18F-florbetapir PET positive according to the visual interpretation by 3 physicians independently as previously reported.

\section{Statistical Analyses}

Quantitative variables were expressed as mean and standard deviation (SD). Categorical variables were expressed as percentage (\%). Differences between the groups were assessed by one-way analysis of variance (ANOVA) or Chi-squared analysis according to the characteristics of data. Post hoc pairwise comparisons of plasma biomarkers between groups were assessed using the least significant difference (LSD) test. Pearson correlation analysis was used to evaluate the relationships between plasma biomarkers and values of $18 \mathrm{~F}$ florbetapir PET SUVR. Receiver operating characteristic (ROC) curve analyses were carried out to evaluate the capacity of plasma biomarkers in discriminating subjects with high composite 18F-florbetapir PET SUVR and subjects diagnosed with AD. All hypothesis testing was twosided, and the level of significance was set at $a=0.05$. Statistical analyses were conducted using IBM SPSS Statistics 23.0 and MedCalc 19.1 . A graphics package (GraphPad Prism, version 8.0) was used to create figures.

\section{Results}

\section{Demographic and Clinical Characteristics}

The demographic and clinical characteristics in the groups of NC, SCD, Obj-SCD, MCl and AD are shown in Table 1. Compared to other groups, subjects with AD were significantly older and had a lower education level. There was no significant difference of gender, diabetes and hypertension prevalence among the groups. Performances on all the neuropsychological tests gradually got worse along with the aggravation of cognitive impairment. Of the 132 subjects who underwent cerebral amyloid scans, the A PET positive rates for each group were $7.6 \%$ $(2 / 26, \mathrm{NC}), 13.7 \%(4 / 29, \mathrm{SCD}), 36.0 \%$ (9/25, Obj-SCD), 48.5\% (17/35, MCI) and $94.1 \%(16 / 17, \mathrm{AD})$ respectively.

\section{Plasma t-tau, $A \beta 40, A \beta 42$ and $A \beta 42 / A \beta 40$ among diagnostic groups}

The plasma levels of t-tau, $A \beta 40, A \beta 42$ and the ratio of $A \beta 42 / A \beta 40$ in different diagnostic groups were shown in Table 2 and Figure 1 . There were significant differences of plasma $A \beta 40, A \beta 42$ and $A \beta 42 / A \beta 40$ ratio among the groups $(P=0.021, P<0.001, P=0.007$, respectively), though no significant difference was found for plasma t-tau ( $P=0.762)$. Post hoc analyses indicated that plasma $A \beta 40$ was significantly higher in SCD and Obj-SCD than in NC (P= 0.0101 and 0.0105) and $A D(P=0.0326$ and 0.0313$)$, while no significant difference was found between NC, $\mathrm{MCl}$ and $\mathrm{AD}$. Plasma $A \beta 42$ was significantly higher in SCD and Obj-SCD than in NC (P=0.0006 and 0.0058$), \mathrm{MCl}(\mathrm{P}=0.0042$ and 0.0262$)$ and $A D$ (both $P<0.0001$ ), while no significant difference was found between $N C$ and $M C l$. Significantly lower plasma $A \beta 42$ and $A \beta 42 / A \beta 40$ ratio was observed in $A D$ than all other groups, while no significant difference of $A \beta 42 / A \beta 40$ ratio was found among NC, SCD, Obj-SCD and MCI.

\section{Associations between plasma biomarkers and amyloid PET}

Associations between plasma biomarkers and SUVR values of amyloid PET were first evaluated in all the subjects who underwent 18Fflorbetapir PET scan. As a result, though no significant correlation was found between plasma t-tau, A 40 and $18 \mathrm{~F}-\mathrm{florbetapir}$ SUVR, plasma $A \beta 42$ and $A \beta 42 / A \beta 40$ ratio were significantly associated with 18F-florbetapir SUVR ( $r=-0.272, P=0.003 ; r=-0.211, P=0.021$ respectively) (Figure

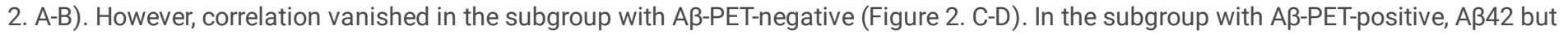
not $A \beta 42 / A \beta 40$ ratio was still correlated with $18 F-$ florbetapir SUVR ( $r=-0.315, P=0.045$ ) (Figure 2 . E-F). In view of plasma $A \beta 40$ and $A \beta 42$ showed bidirectional character along with aggravation of cognitive impairment in our study, we further explored the levels of plasma biomarkers in subgroups with different cerebral A $\beta$ burden. 18F-florbetapir SUVR were classified as low SUVR ( $<1.20)$, moderate SUVR (1.20 to 1.35) and high SUVR (>1.35) according to the results of ROC analyses for SUVR in discriminating between A $\beta$-PET-positive and A 3 -PETnegative (1.20 and 1.35 for specificity $100 \%$ in identifying negative and positive A $\beta$-PET respectively). As shown in Table 3 , plasma $A \beta 42$ and A 42 /A 40 ratio were significantly lower in the subgroup of high SUVR than low and moderate SUVR (All P<0.05). Although not statistically significant, plasma $A \beta 40$ and $A \beta 42$ were both showed an initially increasing trend from low SUVR subgroup to moderate SUVR subgroup, and then decreased in high SUVR subgroup.

\section{Plasma biomarkers as predictors of high brain $A \beta$ burden and $A D$ diagnosis}


In a subset of individuals who underwent both amyloid PET and plasma biomarkers, ROC Analyses were carried out to evaluate the performances of plasma t-tau, $A \beta 40, A \beta 42$ and $A \beta 42 / A b 40$ ratio in predicting high brain $A \beta$ burden (18F-florbetapir SUVR $>1.35$ ). As shown in Figure 3. $A$, the highest value of area under the ROC curve (AUC) was seen with $A \beta 42 / A \beta 40$ ratio (AUC=0.762), followed by plasma $A \beta 42$ $(A U C=0.677)$, though relatively low values were observed with plasma t-tau and $A \beta 40$ (AUC=0.555 and 0.529 respectively).

Comparative diagnostic performances of plasma biomarkers in identifying $A D$ were carried out in all participants regardless of the results of cerebral $A \beta$ status first (Figure 3.B). ROC curves demonstrated the diagnostic accuracy of plasma $A \beta 42(A U C=0.714)$ and $A \beta 42 / A \beta 40$ ratio $(A U C=0.706)$ in discriminating $A D$ from non- $A D$, though plasma t-tau and $A \beta 40$ showed relatively poor performances $(A U C=0.613$ and 0.563 respectively). Furthermore, in subjects with $18 \mathrm{~F}$-florbetapir PET positive, both plasma $A \beta 42(A U C=0.728)$ and $A \beta 42 / A \beta 40$ ratio $(A U C=0.808)$ showed even higher diagnostic accuracy in identifying $A D$, though no significant difference was found when compared to their performances in all subjects (Figure 3.C).

\section{Discussion}

The primary objective of this study was to compare plasma $A \beta 40, A \beta 42$ and t-tau examined by Simoa immunoassay in a continuum of cognitive decline including NC, SCD, Obj-SCD, MCI and AD. Consistent with the previous reports $(6,11,12)$, concentration of plasma $A \beta 42$ and ratio of $A \beta 42 / A \beta 40$ were significantly lower in $A D$ patients, while plasma $A \beta 40$ was less distinct. Plasma t-tau showed no significant difference among the diagnostic groups, which was in agreement with the findings in the cohort of BioFINDER(34). It is worth noting that levels of plasma $A \beta 40$ and $A \beta 42$ were obviously higher in our groups of SCD and Obj-SCD, and showed a significant trend of initially increasing from NC to SCD and Obj-SCD, and then decreasing from SCD and Obj-SCD to MCI and AD. To a great extent, this change was similar to the findings that levels of plasma $A \beta$ isoforms manifested as a bidirectional character over the course of cognitive decline, which was more likely to increase in the early stages of cognitive impairment and decrease prior to clinical AD onset(16-18). As in the Australian Imaging, Biomarkers and Lifestyle (AIBL) study of aging, plasma A 40 and A 42 levels measured by double sandwich ELISA tended to be higher for $\mathrm{MCl}$ than both $\mathrm{NC}$ and $\mathrm{AD}$, though were statistically less significant (16). However, with more detailed grouping criteria for cognitive decline, our results indicated that plasma A 42 and A 440 were significantly higher in SCD and Obj-SCD but not MCI. This may be partly due to the diagnosis of $\mathrm{MCl}$ in this study were based on an actuarial method with comprehensive neuropsychological tests, which may lead to less false positive and greater percentage of progression to dementia compared to conventional $\mathrm{MCl}$ diagnostic criteria(29). Compared to the marginal significance in AIBL, significant differences of plasma $A \beta 40$ and $A \beta 42$ between diagnostic groups in this study may also attribute to the ultrasensitive performance of SIMOA platform we used. On the other hand, given the bidirectional character of plasma $A \beta 40$ and $A \beta 42$ during the progression of cognitive decline in our study, no significant difference of plasma $A \beta 40$ and $A \beta 42$ between the groups of NC and $\mathrm{MCl}$ was not surprising, as observed in this study and previous report(35). In contrast, this study showed no biphasic evolution of $A \beta 42 / A \beta 40$ ratio across the cognitive decline but significantly lower $A \beta 42 / A \beta 40$ ratio in $A D$ than all other groups. At this point, though the relative ratio of $A \beta 42$ and $A \beta 40$ may normalize the pre-analytical variability and eliminate the inter-individual differences for total $A \beta$ concentrations(36, 37$)$, as plasma $A \beta 42$ and $A \beta 40$ showed synchronous changes in SCD and Obj-SCD, the change of $A \beta 42 / A \beta 40$ ratio would inevitably be weakened.

Previous studies demonstrated that plasma amyloid- $\beta$ examined using the Simoa immunoassay could be useful as a potential surrogate for brain $A \beta$ pathology, though the performances were not sufficient and discrepancies remained $(6,7,9,38,39)$. In the present study, relationships between plasma amyloid- $\beta$ and 18F-florbetapir SUVR were assessed not only in all the subjects with A -PET scan, but also in subjects with different $A \beta$-PET status. As a result, both plasma $A \beta 42$ and $A \beta 42 / A \beta 40$ ratio were significantly corelated with $18 F-f l o r b e t a p i r$ SUVR in all the subjects, though the correlation became weak in the subgroup of A of A $\beta$-PET-negative. We therefore speculate that significant correlation between plasma amyloid- $\beta$ and $18 \mathrm{~F}$-florbetapir SUVR in all the subjects may due to their marked alteration between the subgroups of cerebral A $\beta$ positive and negative. In view of the bidirectional character of amyloid- $\beta$ with cognitive decline in our study, we further compared the levels of plasma biomarkers between different cerebral $A \beta$ burden subgroups but not the simple status of positive or negative via visual interpretation of Aß-PET. As a result, similar to their bidirectional character in the progression of cognitive decline, the levels of plasma A 42 and A 40 showed an increasing trend from low SUVR to moderate SUVR and decreasing from moderate SUVR to high SUVR, though only the decreasing of plasma A 42 in the subgroup of high $18 \mathrm{~F}-$ florbetapir SUVR had statistical significance. Taken together, as transportation of $A \beta$ across blood-brain barrier (BBB) plays a major role in $A \beta$ clearance(40), the bidirectional trend of plasma amyloid- $\beta$ along with cognitive decline and cerebral $A \beta$ burden may be associated with the alteration of equilibrium between amyloid- $\beta$ production and clearance in AD continuum. A higher level of amyloid- $\beta$ in peripheral blood may be attributed to the compensatory increased transportation of amyloid- $\beta$ across blood-brain-barrier. At this point, though it is typically considered that decreased $A \beta$ clearance contribute to the predominant pathogenesis of late-onset AD (LOAD)(41), the elevated plasma A 440 and $A \beta 42$ in SCD and Obj-SCD, or not decreased plasma $A \beta 40$ and $A \beta 42$ in the group of moderate A $\beta$-PET SUVR indicated that amyloid- $\beta$ clearance from brain by the BBB was not initially impaired, at least in preclinical AD. However, plasma amyloid- $\beta$ showed significantly decreased with disease progression, consistent with the change in CSF. This may, at least in part, be due to the dysfunction of clearance systems for removing A $\beta$ from brain, such as BBB clearance, Interstitial fluid bulk-flow clearance and CSF absorption clearance(40). In

Page 5/14 
addition, this can also be explained by the acceleration of $A \beta$ aggregation in brain(42), rather than an intrinsic defect of clearance system. On the other hand, whether the initially elevated level of plasma A $\beta$ in SCD and Obj-SCD represents more soluble forms of amyloid- $\beta$ in brain and be a potential window period for anti-A $\beta$ immunotherapy still need more studies to confirm. Either way, the ratio of $A \beta 42 / A \beta 40$ showed a gradually decreasing but not bidirectional trend with the increasing of SUVR. This may contribute to the fact that Aß42 is more likely to form hard-to-clear aggregates than $A \beta 40$ in the progression of cerebral amyloid deposition according to their structural characteristics(43).

Owning to the invasive procedure of CSF and high cost of PET image, blood-based assessments with comparable accuracy in predicting cerebral pathology of $A D$ are urgently needed, especially in population screening. In our current study, a lower ratio of plasma $A \beta 42 / A \beta 40$ demonstrated acceptable value ( $A U C=0.762$ ) in identifying high brain $A \beta$ burden. This was similar to the study in which amyloid-PET positivity was defined by composite SUVR $(A U C=0.79)(38)$, and higher than the study in which amyloid-PET positivity was defined by visually read $(A U C=0.68)(7)$. In any case, with the ultrasensitive technology of Simoa, plasma amyloid- $\beta$ may be a potential surrogate in predicting cerebral $A \beta$ deposition and could undoubtedly serve as a prescreening method to reduce the need of invasive or expensive methods such as lumbar puncture or PET scanning. In our whole cohort regardless the underlying $A \beta$ pathology, both plasma $A \beta 42$ and $A \beta 42 / A \beta 40$ ratio showed potential ability in discriminating the diagnosis of $A D$ from non-AD. Furthermore, in the subset of $A D$ continuum with $A \beta$ positive determined by $18 \mathrm{~F}$-florbetapir PET, both plasma $A \beta 42$ and $A \beta 42 / A \beta 40$ ratio showed even higher diagnostic accuracy in identifying $A D$. Thus, it can be seen that, even $A \beta$ deposition reaches the threshold of positivity in PET imaging, plasma amyloid- $\beta$ may continue to change along with cerebral $A B$ deposition during the natural course of $A D$.

Several limitations should be noted in this study. First, only parts of our participants underwent 18F-florbetapir PET scan, which may have led to the non-significant differences of plasma amyloid- $\beta$ between the groups of low and moderate 18F-florbetapir SUVR. By expanding the sample size of our cohort, we would like to re-assess the changes of plasma amyloid- $\beta$ between different cerebral $A \beta$ burdens or different distributions of $A \beta$ in brain regions. In addition, the NC subgroup had relatively lower prevalence of AB-PET positivity in our study than previous reports, though this could contribute to the fact that SCD and Obj-SCD may be regarded as normal controls in previous studies. Second, as another primary neuropathological hallmarks of AD, phosphorylated tau in blood was not measured in this study. Plasma ptau181 examination and cerebral tau PET scan will be carried out in our future studies to further increase our understanding of cerebral and peripheral biomarkers of AD. Third, designed as a cross sectional study, longitudinal data are needed to confirm the evolution of plasma biomarkers along with cognitive decline within our findings.

\section{Conclusions}

The present study demonstrated that plasma $A \beta 42$ and $A \beta 42 / A \beta 40$ ratio were significantly lower in $A D$ than all other groups. In addition, plasma amyloid- $\beta$ was significantly correlated with $18 \mathrm{~F}$-florbetapir PET SUVR and showed potential value in predicting cerebral A $\beta$ deposition and diagnosis of $A D$. Furthermore, plasma $A \beta 40$ and $A \beta 42$ measured by Simoa immunoassay showed a significantly bidirectional trend of initially increasing from $N C$ to $S C D$ and $O b j-S C D$, and then declining to $M C l$ and $A D$. This may contribute to the status that more soluble forms of amyloid- $\beta$ in brain, or compensatory increased clearance of amyloid- $\beta$ from the brain to periphery in preclinical AD. Our findings encourage future longitudinal investigations on the changes of plasma $A \beta$ along with cognitive decline and cerebral amyloid deposition, and to further explore the mechanisms of this bidirectional character of plasma $A \beta$ with disease progression.

\section{Abbreviations}

Aß: Amyloid- $\beta$; AD: Alzheimer's disease; ACE-III-CV: Chinese version of Addenbrooke's Cognitive Examination III; AVLT: Auditory Verbal Learning Test; AFT: Animal Verbal Fluency Test; ANOVA: one-way analysis of variance; AUC: area under the curve; BBB: blood-brain barrier; BNT: Boston Naming Test; CSF: cerebrospinal fluid; ECOG: Everyday Cognition; FAQ: Functional Assessment Questionnaire; LOAD: late-onset AD; MMSE: Mini-Mental State Examination; MoCA-BC: Chinese version of Montreal Cognitive Assessment-Basic; NIA-AA: National Institute on Aging and Alzheimer's Association; NC: cognitively normal controls; MCl: mild cognitive impairment; Obj-SCD: objectively-defined subtle cognitive decline; PET: positron emission tomography; ROC: Receiver operating characteristic; SCD: subjective cognitive decline; STT-A and B: Shape Trail Test Part A and B; SUVR: standard uptake value ratios; Simoa: single-molecule array; t-tau: total tau

\section{Declarations}

\section{Ethics approval and consent to participate}

This study was approved by the Shanghai Jiao Tong University Affiliated Sixth People's Hospital Foundation Ethical Committee. Written informed consent was obtained from all individual participants or their caregivers included in the study.

\section{Consent for publication}


Not applicable

\section{Availability of data and materials}

The datasets used and/or analysed during the current study are available from the corresponding author on reasonable request.

\section{Competing interests}

The authors declare that they have no competing interests.

\section{Funding}

This work was supported by the National Key R\&D Program of China [grant number 2016YFC1306305]; Guangdong Provincial Key S\&T Program [grant number 2018B030336001]; and Clinical Research Plan of SHDC [grant number SHDC2020CR2056B]. All the funding had no role in the study design, collection and analysis of data, or manuscript preparation.

\section{Authors' contributions}

FFP analysed and interpreted the data, and was a major contributor in writing the manuscript. QH contributed to the PET experiments and drafted and revised the manuscript. YW performed the blood sample collection and processing. YFW had a major role in the acquisition of data. YHG had a major role in PET experiments and data analyses. FX assisted in the PET experiments and revised the manuscript. QHG designed and conceptualised the study, and revised the manuscript. All authors read and approved the final version of the paper.

\section{Acknowledgements}

We would like to thank Jing Zhang and Mei-Yu Geng for their help in the SIMOA immunoassays.

\section{Authors' information}

Not applicable.

\section{References}

1. Long JM, Holtzman DM. Alzheimer Disease: An Update on Pathobiology and Treatment Strategies. Cell. 2019;179(2):312-39.

2. Engelborghs S, De Vreese K, Van de Casteele T, Vanderstichele H, Van Everbroeck B, Cras P, et al. Diagnostic performance of a CSFbiomarker panel in autopsy-confirmed dementia. Neurobiol Aging. 2008;29(8):1143-59.

3. Brier MR, Gordon B, Friedrichsen K, McCarthy J, Stern A, Christensen J, et al. Tau and Abeta imaging, CSF measures, and cognition in Alzheimer's disease. Sci Transl Med. 2016;8(338):338ra66.

4. Kaneko N, Nakamura A, Washimi Y, Kato T, Sakurai T, Arahata Y, et al. Novel plasma biomarker surrogating cerebral amyloid deposition. Proc Jpn Acad Ser B Phys Biol Sci. 2014;90(9):353-64.

5. Li D, Mielke MM. An Update on Blood-Based Markers of Alzheimer's Disease Using the SiMoA Platform. Neurol Ther. 2019;8(Suppl 2):7382.

6. Janelidze S, Stomrud E, Palmqvist S, Zetterberg H, van Westen D, Jeromin A, et al. Plasma beta-amyloid in Alzheimer's disease and vascular disease. Sci Rep. 2016;6:26801.

7. Verberk IMW, Slot RE, Verfaillie SCJ, Heijst H, Prins ND, van Berckel BNM, et al. Plasma Amyloid as Prescreener for the Earliest Alzheimer Pathological Changes. Ann Neurol. 2018;84(5):648-58.

8. Nakamura A, Kaneko N, Villemagne VL, Kato T, Doecke J, Dore V, et al. High performance plasma amyloid-beta biomarkers for Alzheimer's disease. Nature. 2018;554(7691):249-54.

9. Vergallo A, Megret L, Lista S, Cavedo E, Zetterberg H, Blennow K, et al. Plasma amyloid beta 40/42 ratio predicts cerebral amyloidosis in cognitively normal individuals at risk for Alzheimer's disease. Alzheimers Dement. 2019;15(6):764-75.

10. Schindler SE, Bollinger JG, Ovod V, Mawuenyega KG, Li Y, Gordon BA, et al. High-precision plasma beta-amyloid $42 / 40$ predicts current and future brain amyloidosis. Neurology. 2019;93(17):e1647-e59.

11. Rembach A, Faux NG, Watt AD, Pertile KK, Rumble RL, Trounson BO, et al. Changes in plasma amyloid beta in a longitudinal study of aging and Alzheimer's disease. Alzheimers Dement. 2014;10(1):53-61.

12. Hanon O, Vidal JS, Lehmann S, Bombois S, Allinquant B, Treluyer JM, et al. Plasma amyloid levels within the Alzheimer's process and correlations with central biomarkers. Alzheimers Dement. 2018;14(7):858-68. 
13. Giudici KV, de Souto Barreto P, Guyonnet S, Li Y, Bateman RJ, Vellas B, et al. Assessment of Plasma Amyloid-beta42/40 and Cognitive Decline Among Community-Dwelling Older Adults. JAMA Netw Open. 2020;3(12):e2028634.

14. Verberk IMW, Hendriksen HMA, van Harten AC, Wesselman LMP, Verfaillie SCJ, van den Bosch KA, et al. Plasma amyloid is associated with the rate of cognitive decline in cognitively normal elderly: the SCIENCe project. Neurobiol Aging. 2020;89:99-107.

15. Chouraki V, Beiser A, Younkin L, Preis SR, Weinstein G, Hansson O, et al. Plasma amyloid-beta and risk of Alzheimer's disease in the Framingham Heart Study. Alzheimers Dement. 2015;11(3):249-57. e1.

16. Lui JK, Laws SM, Li QX, Villemagne VL, Ames D, Brown B, et al. Plasma amyloid-beta as a biomarker in Alzheimer's disease: the AIBL study of aging. J Alzheimers Dis. 2010;20(4):1233-42.

17. Wang J, Qiao F, Shang S, Li P, Chen C, Dang L, et al. Elevation of Plasma Amyloid-beta Level is More Significant in Early Stage of Cognitive Impairment: A Population-Based Cross-Sectional Study. J Alzheimers Dis. 2018;64(1):61-9.

18. Chen TB, Lai YH, Ke TL, Chen JP, Lee YJ, Lin SY, et al. Changes in Plasma Amyloid and Tau in a Longitudinal Study of Normal Aging, Mild Cognitive Impairment, and Alzheimer's Disease. Dement Geriatr Cogn Disord. 2019;48(3-4):180-95.

19. Katzman R, Zhang MY, Ouang Ya Q, Wang ZY, Liu WT, Yu E, et al. A Chinese version of the Mini-Mental State Examination; impact of illiteracy in a Shanghai dementia survey. J Clin Epidemiol. 1988;41(10):971-8.

20. Huang L, Chen KL, Lin BY, Tang L, Zhao QH, Lv YR, et al. Chinese version of Montreal Cognitive Assessment Basic for discrimination among different severities of Alzheimer's disease. Neuropsychiatr Dis Treat. 2018;14:2133-40.

21. Pan FF, Wang Y, Huang L, Huang Y, Guo QH. Validation of the Chinese version of Addenbrooke's cognitive examination III for detecting mild cognitive impairment. Aging Ment Health. 2021:1-8.

22. Farias ST, Mungas D, Reed BR, Cahn-Weiner D, Jagust W, Baynes K, et al. The measurement of everyday cognition (ECog): scale development and psychometric properties. Neuropsychology. 2008;22(4):531-44.

23. Pfeffer RI, Kurosaki TT, Harrah CH Jr, Chance JM, Filos S. Measurement of functional activities in older adults in the community. J Gerontol. 1982;37(3):323-9.

24. Zhao Q, Guo Q, Liang X, Chen M, Zhou Y, Ding D, et al. Auditory Verbal Learning Test is Superior to Rey-Osterrieth Complex Figure Memory for Predicting Mild Cognitive Impairment to Alzheimer's Disease. Curr Alzheimer Res. 2015;12(6):520-6.

25. Guo QH, HZ, Shi WX, Sun YM, Lv CZ. Boston naming test using by Chinese elderly, patient with mild cognitive impairment and Alzheimer's dementia. Journal of Chinese Mental Health. 2006;20:81-5.

26. Zhao Q, Guo Q, Hong Z. Clustering and switching during a semantic verbal fluency test contribute to differential diagnosis of cognitive impairment. Neurosci Bull. 2013;29(1):75-82.

27. Zhao Q, Guo Q, Li F, Zhou Y, Wang B, Hong Z. The Shape Trail Test: application of a new variant of the Trail making test. PLoS One. 2013;8(2):e57333.

28. McKhann GM, Knopman DS, Chertkow H, Hyman BT, Jack CR Jr, Kawas CH, et al. The diagnosis of dementia due to Alzheimer's disease: recommendations from the National Institute on Aging-Alzheimer's Association workgroups on diagnostic guidelines for Alzheimer's disease. Alzheimers Dement. 2011;7(3):263-9.

29. Bondi MW, Edmonds EC, Jak AJ, Clark LR, Delano-Wood L, McDonald CR, et al. Neuropsychological criteria for mild cognitive impairment improves diagnostic precision, biomarker associations, and progression rates. J Alzheimers Dis. 2014;42(1):275-89.

30. Jessen F, Amariglio RE, van Boxtel M, Breteler M, Ceccaldi M, Chetelat G, et al. A conceptual framework for research on subjective cognitive decline in preclinical Alzheimer's disease. Alzheimers Dement. 2014;10(6):844-52.

31. Wilson DH, Rissin DM, Kan CW, Fournier DR, Piech T, Campbell TG, et al. The Simoa HD-1 Analyzer: A Novel Fully Automated Digital Immunoassay Analyzer with Single-Molecule Sensitivity and Multiplexing. J Lab Autom. 2016;21(4):533-47.

32. Greve DN, Salat DH, Bowen SL, Izquierdo-Garcia D, Schultz AP, Catana C, et al. Different partial volume correction methods lead to different conclusions: An (18)F-FDG-PET study of aging. Neuroimage. 2016;132:334-43.

33. Gonzalez-Escamilla G, Lange C, Teipel S, Buchert R, Grothe MJ. Alzheimer's Disease Neuroimaging I. PETPVE12: an SPM toolbox for Partial Volume Effects correction in brain PET - Application to amyloid imaging with AV45-PET. Neuroimage. 2017;147:669-77.

34. Mattsson N, Zetterberg H, Janelidze S, Insel PS, Andreasson U, Stomrud E, et al. Plasma tau in Alzheimer disease. Neurology. 2016;87(17):1827-35.

35. Palmqvist S, Janelidze S, Stomrud E, Zetterberg H, Karl J, Zink K, et al. Performance of Fully Automated Plasma Assays as Screening Tests for Alzheimer Disease-Related beta-Amyloid Status. JAMA Neurol. 2019.

36. Wiltfang J, Esselmann $\mathrm{H}$, Bibl M, Hull M, Hampel $\mathrm{H}$, Kessler $\mathrm{H}$, et al. Amyloid beta peptide ratio $42 / 40$ but not A beta 42 correlates with phospho-Tau in patients with low- and high-CSF A beta 40 load. J Neurochem. 2007;101(4):1053-9. 
37. Willemse E, van Uffelen K, Brix B, Engelborghs S, Vanderstichele H, Teunissen C. How to handle adsorption of cerebrospinal fluid amyloid beta (1-42) in laboratory practice? Identifying problematic handlings and resolving the issue by use of the Abeta42/Abeta40 ratio. Alzheimers Dement. 2017;13(8):885-92.

38. De Meyer S, Schaeverbeke JM, Verberk IMW, Gille B, De Schaepdryver M, Luckett ES, et al. Comparison of ELISA- and SIMOA-based quantification of plasma Abeta ratios for early detection of cerebral amyloidosis. Alzheimers Res Ther. 2020;12(1):162.

39. Tanaka T, Ruifen JC, Nai YH, Tan CH, Lim CZJ, Zhang Y, et al. Head-to-head comparison of amplified plasmonic exosome Abeta42 platform and single-molecule array immunoassay in a memory clinic cohort. Eur J Neurol. 2021;28(5):1479-89.

40. Tarasoff-Conway JM, Carare RO, Osorio RS, Glodzik L, Butler T, Fieremans E, et al. Clearance systems in the brain-implications for Alzheimer disease. Nat Rev Neurol. 2015;11(8):457-70.

41. Mawuenyega KG, Sigurdson W, Ovod V, Munsell L, Kasten T, Morris JC, et al. Decreased clearance of CNS beta-amyloid in Alzheimer's disease. Science. 2010;330(6012):1774.

42. Potter R, Patterson BW, Elbert DL, Ovod V, Kasten T, Sigurdson W, et al. Increased in vivo amyloid-beta42 production, exchange, and loss in presenilin mutation carriers. Sci Transl Med. 2013;5(189):189ra77.

43. Lin Y, Im H, Diem LT, Ham S. Characterizing the structural and thermodynamic properties of Abeta42 and Abeta40. Biochem Biophys Res Commun. 2019;510(3):442-8.

\section{Tables}


Table 1. Demographics, neuropsychological tests and 18F-florbetapir PET imaging for NC, SCD, Obj-SCD, MCI and AD

\begin{tabular}{|c|c|c|c|c|c|c|}
\hline Index & $N C(n=76)$ & $\operatorname{SCD}(n=77)$ & Obj-SCD $(n=61)$ & $\mathrm{MCl}(\mathrm{n}=92)$ & $\mathrm{AD} \otimes n=101 \rrbracket$ & $\begin{array}{l}F / x^{2}(P \\
\text { value })\end{array}$ \\
\hline
\end{tabular}

\section{Demographics}

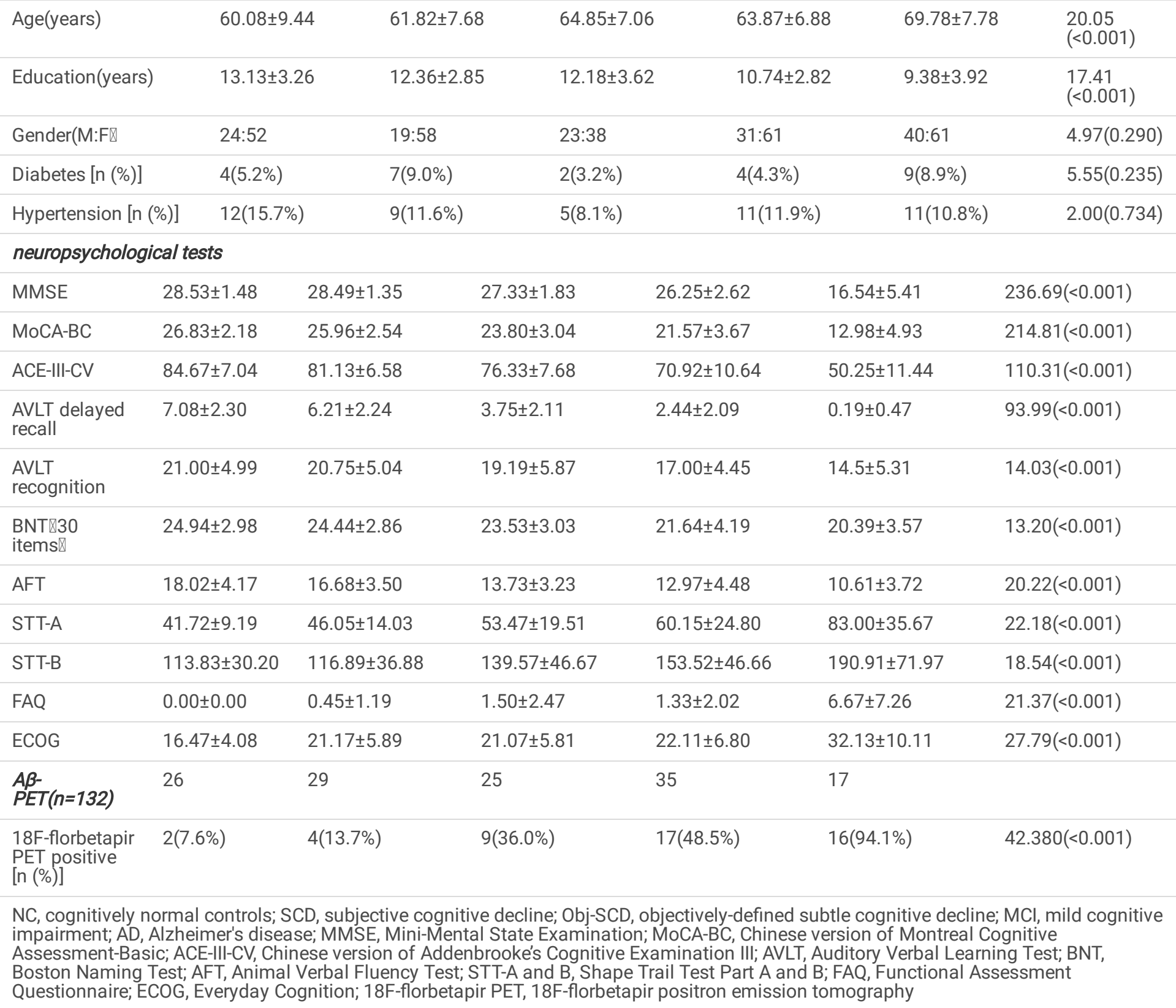


Table 2. Plasma t-tau, $A \beta 40, A \beta 42$ and $A \beta 42 / A \beta 40$ for NC, SCD, Obj-SCD, MCl and AD

\begin{tabular}{|c|c|c|c|c|c|c|c|}
\hline Plasma biomarkers & $N C(n=7$ & $\operatorname{SCD}(n=77$ & Obj-S & $=61)$ & $\mathrm{MCl}(\mathrm{n}=92)$ & $A D \bigotimes n=101 \rrbracket$ & $\mathrm{F}$ (P value) \\
\hline T-tau (pg/ml) & $1.46 \pm 1.82$ & $1.60 \pm 1.75$ & $1.43 \pm 1.56$ & 1.41 & & $1.67 \pm 1.16$ & $0.464(0.762)$ \\
\hline $\mathrm{A} \beta 40(\mathrm{pg} / \mathrm{ml})$ & $137.74 \pm 42.25$ & $160.04 \pm 49.54$ & $162.33 \pm 44.23$ & 144. & 8.47 & $142.76 \pm 61.38$ & $2.932(0.021)$ \\
\hline $\mathrm{A} \beta 42(\mathrm{pg} / \mathrm{ml})$ & $9.22 \pm 2.69$ & $10.90 \pm 2.84$ & $10.72 \pm 3.07$ & 9.56 & & $7.90 \pm 2.89$ & $13.870(<0.001)$ \\
\hline$A \beta 42 / A \beta 40$ (ratio) & $0.0697 \pm 0.0177$ & $0.0712 \pm 0.0165$ & $0.0690 \pm 0.0177$ & 0.0 & 0.0141 & $0.0613 \pm 0.0254$ & $3.605(0.007)$ \\
\hline
\end{tabular}

NC, cognitively normal controls; SCD, subjective cognitive decline; Obj-SCD, objectively-defined subtle cognitive decline; MCI, mild cognitive impairment; $A D$, Alzheimer's disease; $A \beta$, Amyloid- $\beta$

\begin{tabular}{|c|c|c|c|c|}
\hline \multirow[t]{2}{*}{ Index } & \multicolumn{3}{|c|}{ 18F-florbetapir PET } & \multirow[t]{2}{*}{ F (P value) } \\
\hline & SUVR $<1.2$ & $1.2 \leq$ SUVR<1.35 & SUVR $\geq 1.35$ & \\
\hline T-tau (pg/ml) & $1.57 \pm 2.29$ & $1.66 \pm 2.07$ & $1.71 \pm 2.27$ & $0.037(0.964)$ \\
\hline $\mathrm{A} \beta 40(\mathrm{pg} / \mathrm{ml})$ & $131.50 \pm 50.90$ & $143.02 \pm 53.74$ & $134.89 \pm 45.07$ & $0.612(0.544)$ \\
\hline$A \beta 42(p g / m l)$ & $9.54 \pm 3.45$ & $9.90 \pm 3.50$ & $7.76 \pm 2.59 *+$ & $3.621(0.030)$ \\
\hline$A \beta 42 / A \beta 40$ (ratio) & $0.0776 \pm 0.0221$ & $0.0732 \pm 0.0201$ & $0.0615 \pm 0.0177^{*}+$ & $4.657(0.011)$ \\
\hline \multicolumn{5}{|c|}{ 18F-florbetapir PET, 18F-florbetapir positron emission tomography; $A \beta$, Amyloid- $\beta$; SUVR, standard } \\
\hline \multicolumn{5}{|c|}{ Comparations between $1.2 \leq S U V R<1.35$ and $S U V R \geq 1.35$ are marked behind $S U V R \geq 1.35$, $\dagger$ for $P<0.05$} \\
\hline
\end{tabular}

\section{Figures}



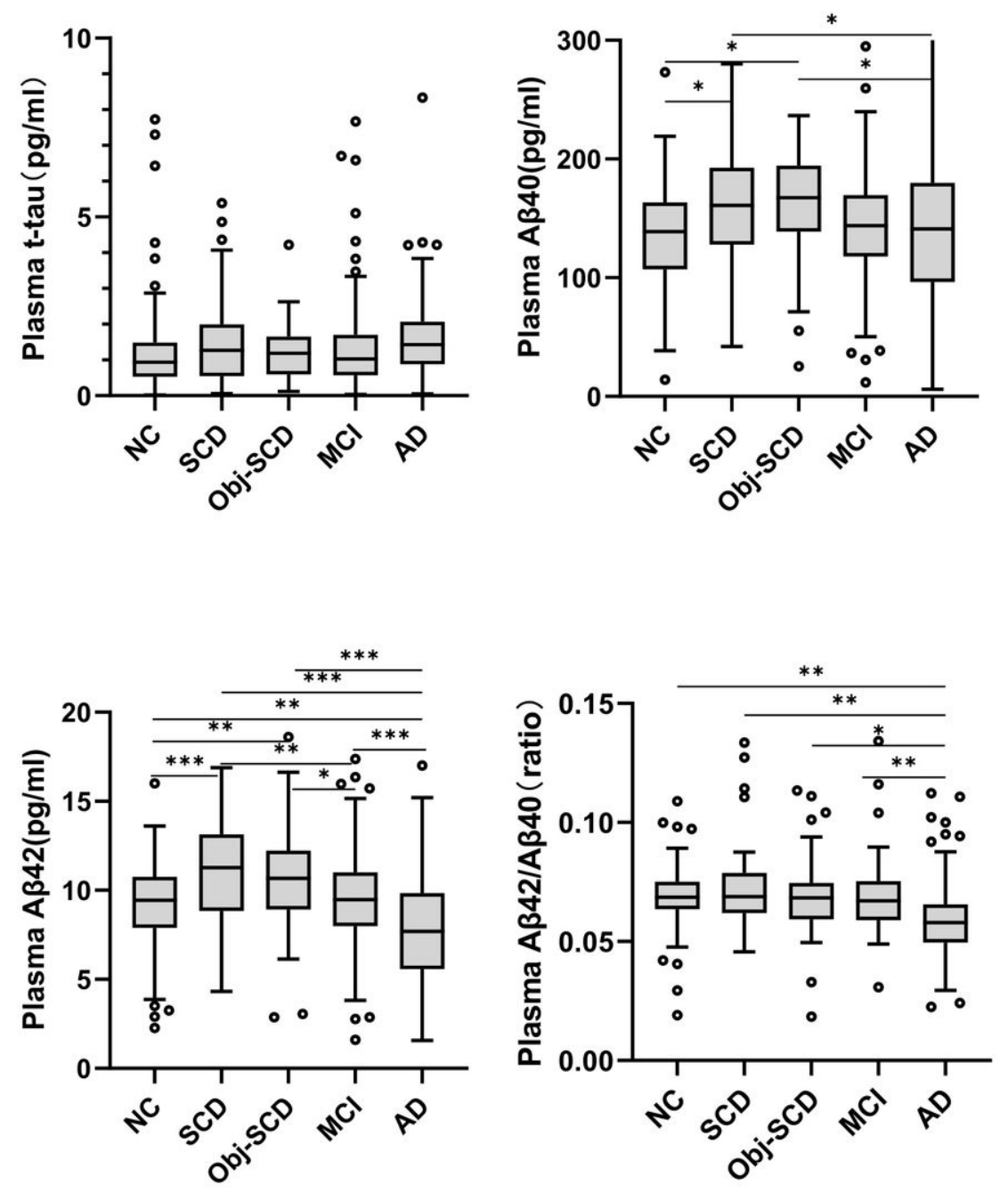

Figure 1

Comparisons of plasma biomarkers among the groups of cognitively normal controls (NC), subjective cognitive decline (SCD), objectivelydefined subtle cognitive decline (Obj-SCD), mild cognitive impairment $(\mathrm{MCl})$ and Alzheimer's disease (AD). ${ }^{*} \mathrm{P}<0.05 ; \star \star \mathrm{P}<0.01 ; * \star * P<0.001$ 

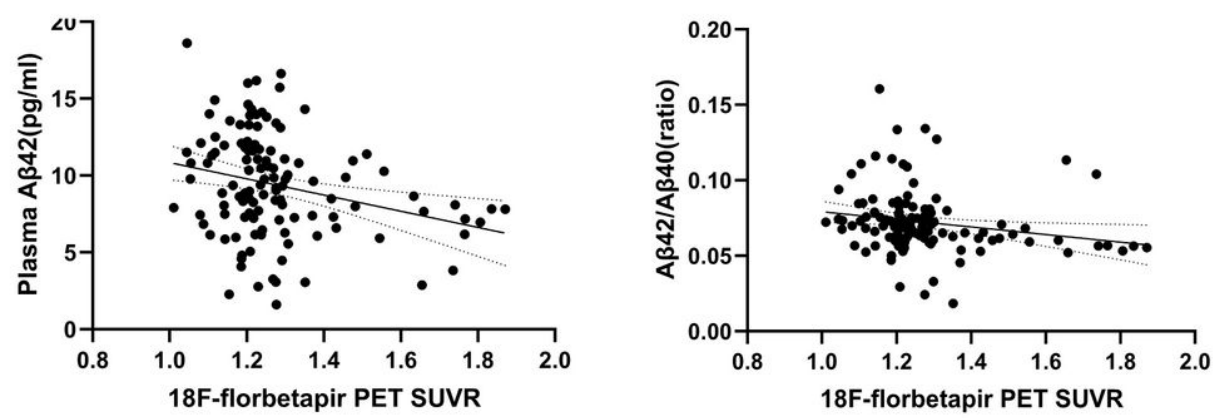

c.

$r=-0.188 P=0.098$

D.

$r=-0.154 P=0.178$
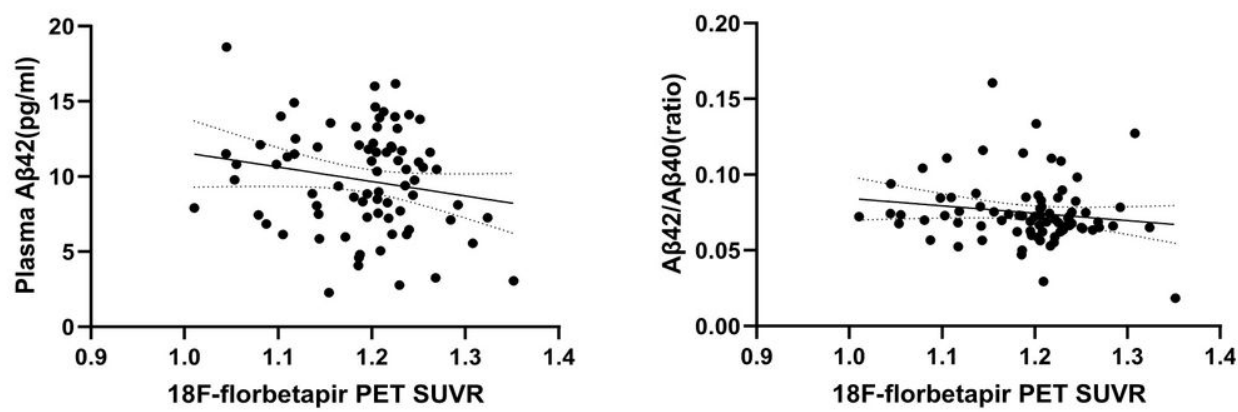

E.

$r=-0.315 \quad P=0.045$

F.

$r=-0.114 P=0.479$
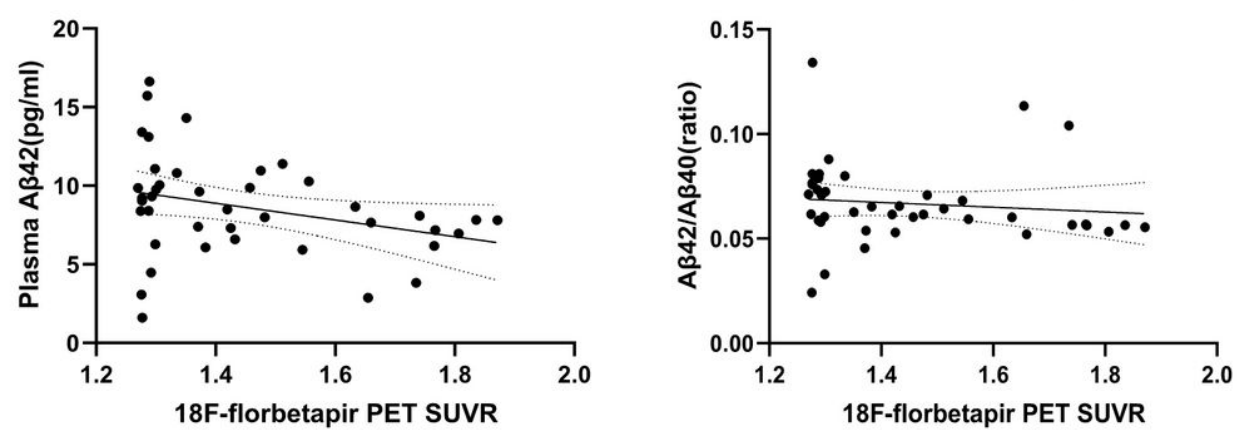

Figure 2

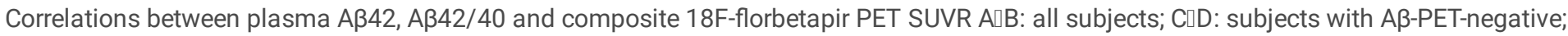

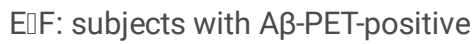

A.

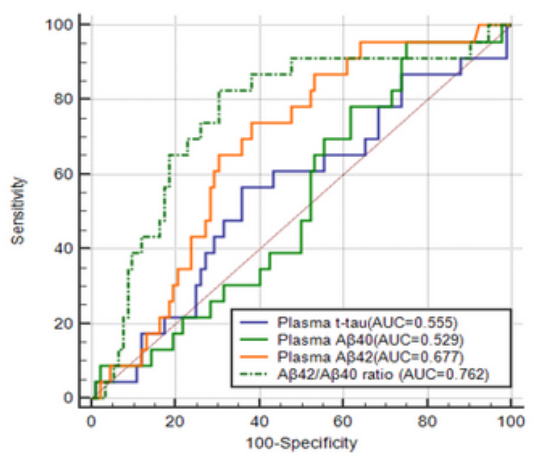

B.

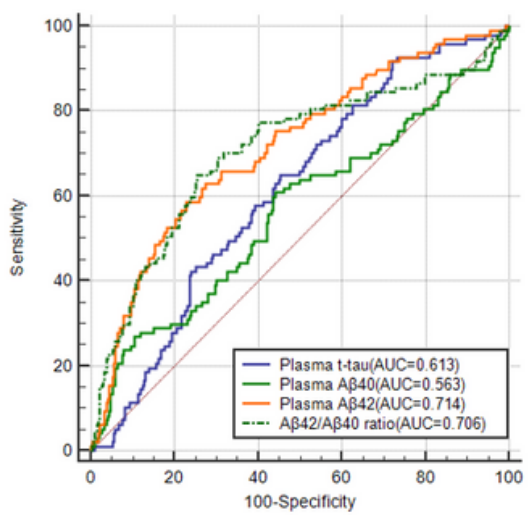

C.

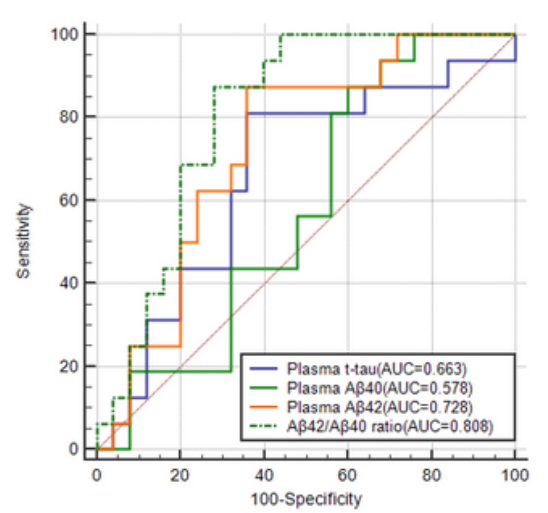

Figure 3 
A. ROC curves for plasma $A \beta 42$ and $A \beta 42 / 40$ in discriminating composite $18 F$-florbetapir PET SUVR $\geq 1.35$ from <1.35; $B$. ROC curves for plasma $A \beta 42$ and $A \beta 42 / 40$ in discriminating $A D$ from non-AD; $C$. ROC curves for plasma $A \beta 42$ and $A \beta 42 / 40$ in discriminating $A D$ from non- $A D$ in subjects with $A \beta$-PET-positive 